\title{
Improvement of the finite element method equations conditioning for the magnetic field-circuital problems
}

\author{
MAREK GOLĘBIOWSKI \\ Faculty of Electrical and Computer Engineering, Rzeszow University of Technology \\ Wincentego Pola 2, 35-959 Rzeszów, Poland \\ e-mail:yegolebi@prz.edu.pl
}

(Received: 16.09.2016, revised: 16.01.2017)

\begin{abstract}
The presented systems with magnetically coupled windings are solved with the finite element method. If the issue of voltage supply is analyzed, a system of linear equations with a partially skew-symmetric sparse matrix is obtained. Iterative methods used to solve a system of equations are particularly effective for symmetric matrices. Resultant equations can be reduced to this symmetrical form by using the method known from the literature [1]. The ratio of the maximum to the minimum eigenvalue of the main matrix of this circuit, which is the condition number, is however very high. This means that the problem is ill-conditioned and leads to a very long iterative solution process. The method presented in the article allows for a direct solution of a system of equations on its part, corresponding to high eigenvalues of the system matrix. The remaining part of the system of equations is solved by iterative methods. This part has much better condition number, and therefore the computational process is fast. The proposed iterative process depends on multiplication of a sparse matrix by vectors. It is not necessary (and possible) to store the entire matrix. This is especially important for larger sizes of a matrix.
\end{abstract}

Key words: magnetic field-circuital problem, condition number, iterative method

\section{Introduction}

In many problems a large linear system of algebraic equations of the form (1) must be solved in the following way:

$$
[A] \cdot \overrightarrow{\boldsymbol{x}}=\overrightarrow{\boldsymbol{b}} ; \overrightarrow{\boldsymbol{r e s}}=[A] \cdot \overrightarrow{\boldsymbol{x}}-\overrightarrow{\boldsymbol{b}},
$$

where: $\overrightarrow{\boldsymbol{b}}$ is a known vector, $[\boldsymbol{A}]$ is a square, symmetrical, positive definite $n \times n$ matrix, $\overrightarrow{\boldsymbol{r e s}}$ is the error of the $\overrightarrow{\boldsymbol{x}}$ solution.

We meet often in reality with the issue of this form, e.g. with boundary problems of partial differential equations of elliptic type, when designing a network, trusses, etc. Due to these issues the dimension $n$ is so large that the overall number of elements in the matrix $n^{2}$ greatly exceeds the capabilities of a digital machine's memory.

Therefore, one can use the special form of the matrix $[A]$, which is usually a sparse matrix $[1,2]$. The efficient iterative methods of solving problems of type (1) were developed. The 
best of these methods, e.g. a Chebyshev method requires certain additional information about the spectrum of the matrix $[A][2,3]$.

The minimum information is the knowledge of the smallest possible range containing the spectrum (eigenvalues) of the matrix $[\boldsymbol{A}]$. This information is not always available. On the other hand, there are methods like e.g. the conjugate gradient method or minimal residual method, which do not require any information about the spectrum of the matrix $[A]$.

These methods, however, are unstable. What is noteworthy is an idea to combine two methods: the Chebyshev method with the minimal residual method [2,3]. The essence of this combination is alternating the work of these methods. The Chebyshev method reduces errors of the solution $\overrightarrow{\boldsymbol{x}}$ of the system (1) for high eigenvalues of the matrix [A]. The minimal residual method reduces errors of the solution corresponding with small eigenvalues of the matrix $[A]$. As soon as the minimal residual method loses stability, the error norm, i.e. the norm of the residuum:

$$
\overrightarrow{\boldsymbol{r e s}}=[A] \cdot \overrightarrow{\boldsymbol{x}}-\vec{b}
$$

is enlarged.

Then the stable Chebyshev method that reduces errors associated with the upper half of the spectrum of the matrix $[\boldsymbol{A}]$ is executed [4]. The greatest eigenvalue of $\lambda_{\max }$ is usually easy to estimate, e.g. using the Gershgorin theorem [5], or by adopting any norm of the matrix $[A]$ corresponding to the vector norm.

It is assumed that the Chebyshev method is intended to reduce errors of a solution corresponding to the eigenvalues from the range of

$$
\left[\lambda_{\max }, \frac{1}{2} \lambda_{\max }\right] .
$$

The alternative use of Chebyshev methods and the minimal residual method $[3,4]$ always allows to obtain a solution of the symmetrical system of Equations (1).

The essence of the proposal included in this article is to replace the Chebyshev method by a direct solution (i.e. not iterative). This step resets the errors associated with the upper part of the spectrum of the matrix $[A]$ in a single execution. Further reduction of residuum will be performed by, also previouslv initiated, the iterative minimal residual method.

A part of the solution $\overrightarrow{\boldsymbol{x}}$ associated with smaller eigenvalues of the spectrum of the matrix $[\boldsymbol{A}]$ will be improved with this iterative method. The ratio of the maximum eigenvalue to the minimum eigenvalue of this spectrum part will be lower than in full spectrum of the matrix $[\boldsymbol{A}]$. This means better conditioning of the minimal residual method and a much lower number of iterations needed.

\section{The finite element method equations for the systems with magnetically coupled windings}

The magnetic field is expressed by a vector potential 


$$
\overrightarrow{\boldsymbol{A}}=\sum_{i} \vec{N}_{\boldsymbol{i}} \cdot A_{i},
$$

where $\vec{N}_{i}$ represents the edge basis functions. Scalar potential is expressed by nodal basis functions $N_{i}$ as

$$
\varphi=\sum_{i} N_{i} \cdot \varphi_{i}
$$

The current density of the $j$-th winding with the current $i$, i.e. $\overrightarrow{\boldsymbol{J}}_{j}$ is expressed by the vector introduced by $\overrightarrow{\boldsymbol{T}}_{j}$ as $\overrightarrow{\boldsymbol{J}}_{j}=\overrightarrow{\operatorname{rot}}\left(\overrightarrow{\boldsymbol{T}}_{j}\right) \cdot i_{j} \quad[1,6,9]$. The vector $\overrightarrow{\boldsymbol{T}}_{j}$ is stretched on a $j$-th winding. By its means, one can write the magnetic flux coupled with $j$-th winding:

$$
\Psi_{j}=\int_{V} \overrightarrow{\boldsymbol{T}}_{j} \cdot \overrightarrow{\boldsymbol{B}} \mathrm{d} V=\int_{V} \overrightarrow{\boldsymbol{T}}_{j} \cdot \overrightarrow{\operatorname{rot}}(\overrightarrow{\boldsymbol{A}}) \mathrm{d} V
$$

where $\vec{B}$ is the magnetic induction.

Voltage equations for windings can be written (after integrating) as:

$$
\Psi_{j}+L_{\sigma j} i_{j}+R_{j} \int_{0}^{t} i \mathrm{~d} t=\int_{0}^{t} u_{j} \mathrm{~d} t ; j=1,2, \ldots f,
$$

where $L_{\sigma j}$ is the leakage inductance of the $j$-th winding, $R_{j}$ is its resistance, $u_{j}$ is the supplying voltage, and $f$ is a number of phases (of windings).

In $2 \mathrm{D}$ calculations, leakage inductance of the winding $L_{\sigma j}$ should include the endwinding and mains leakage inductance. With the use of implicit methods of solving equations, $L_{\sigma j}+R_{j} / 2 \mathrm{~d} t$ should be considered as leakage inductance, where $\mathrm{d} t$ is the integration step. In 3D calculations, the end-windings inductance is not included because it is included directly by the finite element method. With $v$ the inverse of magnetic permeability and with $\gamma$ electrical conductivity of metal sheets are determined. Applying the Galerkin weak formulation $[1,9]$ a set of equations of the finite element method for systems with magnetically coupled windings can be written $[1,6,9]$ as:

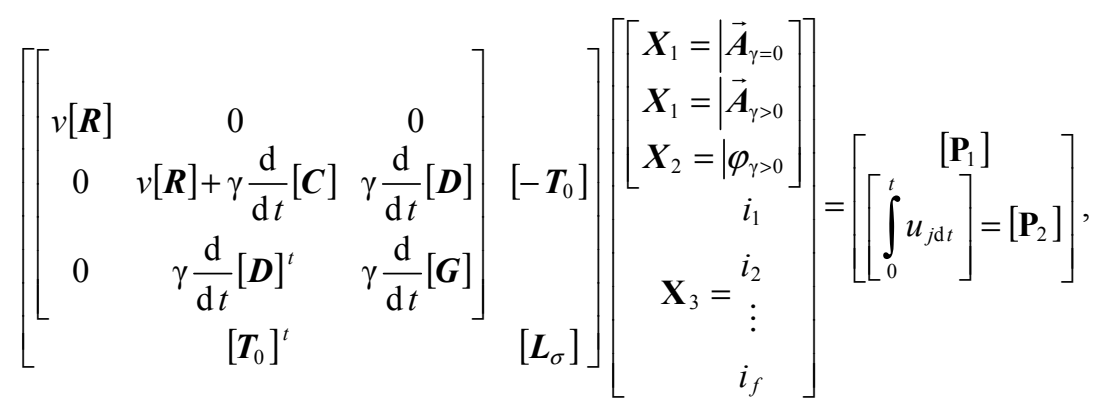

where: 


$$
\begin{gathered}
{[\boldsymbol{R}]_{i j}=\iiint_{V} \overrightarrow{\operatorname{rot}} \overrightarrow{\boldsymbol{N}}_{\boldsymbol{i}} \cdot \overrightarrow{\operatorname{rot}} \overrightarrow{\boldsymbol{N}}_{\boldsymbol{j}} \mathrm{d} V, \quad[\boldsymbol{G}]_{i j}=\iiint_{V} \overrightarrow{\operatorname{grad}} N_{i} \cdot \overrightarrow{\operatorname{grad}} N_{j} \mathrm{~d} V,} \\
{[\boldsymbol{C}]_{i j}=\iiint_{V} \overrightarrow{\boldsymbol{N}}_{\boldsymbol{i}} \cdot \overrightarrow{\boldsymbol{N}}_{\boldsymbol{j}} \mathrm{d} V, \quad[\boldsymbol{D}]_{i j}=\iiint_{V} \overrightarrow{\boldsymbol{N}}_{\boldsymbol{i}} \cdot \overrightarrow{\operatorname{grad}} N_{j} \mathrm{~d} V,} \\
{\left[\boldsymbol{T}_{0}\right]_{\boldsymbol{i} \boldsymbol{j}}=\iiint_{V} \overrightarrow{\operatorname{rot}} \overrightarrow{\boldsymbol{N}}_{\boldsymbol{i}} \cdot \overrightarrow{\boldsymbol{T}}_{\boldsymbol{j}} \mathrm{d} V,}
\end{gathered}
$$

where: $i$ is the line number, $j$ is the number of windings, and $t$ is the matrix transposition.

In conductive areas, where the electrical conductivity $\gamma \neq 0$ the scalar potentials $\varphi$ are unknowns.

Partial skew-symmetry is related to winding currents, that act as a vector of unknowns $\left[\boldsymbol{X}_{3}\right]$. This is confirmed by Maxwell's equations:

$$
\overrightarrow{\operatorname{rot}} \overrightarrow{\boldsymbol{H}}=\overrightarrow{\boldsymbol{J}} ; \overrightarrow{\operatorname{rot} \overrightarrow{\boldsymbol{E}}}=\frac{-\partial \overrightarrow{\boldsymbol{B}}}{\partial t} .
$$

The resulting Equation (5) with a sparse matrix, due to the large size, should be solved by iterative methods. The symmetry of the matrix radically quickens the solution of iterative methods. It is therefore necessary to bring the system (5) to a symmetrical form [1].

The system of Equations (5) can be written as:

$$
\left[\begin{array}{cc}
M & -T_{0} \\
T_{0}^{t} & L_{\sigma}
\end{array}\right] \cdot\left[\begin{array}{c}
X_{12} \\
X_{3}
\end{array}\right]=\left[\begin{array}{c}
P_{1} \\
P_{2}
\end{array}\right] \text {, where }\left[X_{12}\right]=\left[\begin{array}{l}
X_{1} \\
X_{2}
\end{array}\right] \text {. }
$$

By reducing the unknowns $\left[\boldsymbol{X}_{3}\right]$ from the system, it can be reduced to a symmetrical form of:

$$
\left[M+T_{0} \cdot L_{\sigma}^{-1} \cdot T_{0}^{t}\right] \cdot\left[X_{12}\right]=\left[P_{1}+T_{0} \cdot L_{\sigma}^{-1} \cdot P_{2}\right]=[P]
$$

and

$$
\left[X_{3}\right]=L_{\sigma}^{-1}\left[P_{2}-T_{0}^{t} \cdot\left[X_{12}\right]\right] .
$$

The system of Equations (8) is symmetrical, because the $\left[\boldsymbol{L}_{\boldsymbol{\sigma}}\right]$ matrix is symmetrical. The main matrix of the system includes components created by the finite element method introduced by the matrix $[\boldsymbol{M}]$. It also includes the components associated with $\left[\boldsymbol{L}_{\boldsymbol{\sigma}}\right]^{-1}$ that have high values. Therefore, this matrix spectrum is wide. The ratio of the highest to the lowest eigenvalue, i.e. the condition number is large. This implies a large number of iterations at which the stability of the solution may be lost. It is possible, however, to extract earlier the solution's components associated with large eigenvalues. They are introduced into the system (8) by the element $\left[\boldsymbol{L}_{\boldsymbol{\sigma}}\right]^{-1}$. For this purpose, a direct method is used. It solves a system of small dimensions, because the $\left[\boldsymbol{L}_{\boldsymbol{\sigma}}\right]$ matrix has a size equal to the $f$ number of windings.

This direct method replaces the previously described Chebyshev iterations. This method is the essence of the presented article.

Other components of the solution, corresponding to smaller eigenvalues, are obtained by iterative methods, e.g. by the minimal residual method. They are much faster after the elimi- 
nation of the components corresponding to large eigenvalues. They work in a smaller range of the spectrum, so conditioning is much better. Despite a series of additional operations related therein and large dimension of the issue, iterative calculations can be carried out using the sparse matrix technique.

\section{Improvement of the matrix condition number of the system with magnetically coupled windings}

The reduction of matrix spectrum of the magnetic system will be exemplified by the system solved with circuital methods. The principle of the transformation is the same as for a system solved with the finite element method (5). However, due to smaller dimensions of the equations it is easier to observe the results of transformations. The tested system is shown in Fig. 1.

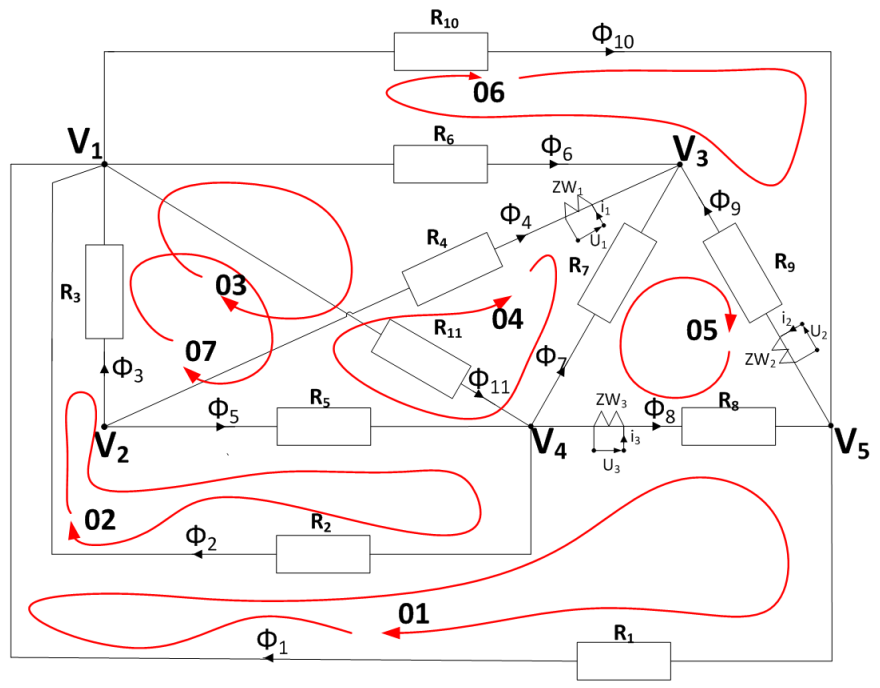

Fig. 1. System with three magnetically coupled windings $(g=11, s=7, r=5)$

An exemplary system has $g=11$ magnetic cores with the $\Phi_{g i}$ magnetic fluxes, $(i=1: 11)$. Magnetic reluctance (resistance) of cores is marked by $R_{i},(i=1: 11)$.

The magnetic system has $r=5$ nodes with magnetic potentials $V_{i},(i=1: 5)$. This means that the system of equations has $s=g-(r-1)=7$ independent loops (degrees of freedom). It will be solved by the loop flux method.

The loop fluxes $\Phi_{o i}(i=1: 7)$ are introduced. The loops are indicated in Fig. 1 as $O_{i},(i=1: 7)$. Connections of cores are described by the connection matrix $\operatorname{pol}(s=7, g=11)$. From the fluxes of loops $\Phi_{O}$ one can obtain fluxes in the branches $\Phi_{g}$.

$$
\left[\boldsymbol{\Phi}_{g}\right]=[\boldsymbol{p o l}]^{t} \cdot\left[\boldsymbol{\Phi}_{O}\right]
$$


where the connection matrix $[\mathrm{pol}]$ is:

$$
[\boldsymbol{p o l}]=\left[\begin{array}{ccccccccccc}
1 & -1 & 0 & 0 & 0 & 0 & 0 & 1 & 0 & 0 & 0 \\
0 & 1 & -1 & 0 & 1 & 0 & 0 & 0 & 0 & 0 & 0 \\
0 & 0 & 1 & -1 & 0 & 1 & 0 & 0 & 0 & 0 & 0 \\
0 & 0 & 0 & 1 & -1 & 0 & -1 & 0 & 0 & 0 & 0 \\
0 & 0 & 0 & 0 & 0 & 0 & 1 & -1 & -1 & 0 & 0 \\
0 & 0 & 0 & 0 & 0 & -1 & 0 & 0 & 1 & 1 & 0 \\
0 & 0 & 1 & 0 & -1 & 0 & 0 & 0 & 0 & 0 & 1
\end{array}\right] .
$$

Magnetic reluctances of cores form a square matrix of the values established on the diagonal are:

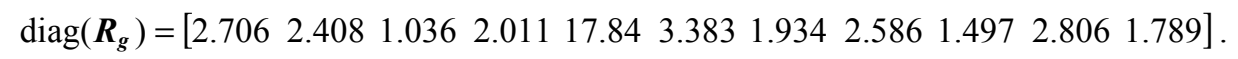

On the magnetic cores with numbers 4,9 and 8 windings were placed with the number of coils of respectively $z w_{1}=2, z w_{2}=1, z w_{3}=4$. Therefore magnetomotive forces can be written as: $\Theta_{4}=z w_{1} \cdot i_{1}, \Theta_{9}=z w_{2} \cdot i_{2}, \Theta_{8}=z w_{3} \cdot i_{3}$, where $i_{1}, i_{2}, i_{3}$ are the winding currents. The remaining cores have specific electric loading equal to zero. Creating a winding number matrix $[\boldsymbol{Z} w]$ with dimension $(g \times f)$ is:

$$
[\boldsymbol{Z} \boldsymbol{w}]=\left[\begin{array}{ccccccccccc}
0 & 0 & 0 & z w_{1} & 0 & 0 & 0 & 0 & 0 & 0 & 0 \\
0 & 0 & 0 & 0 & 0 & 0 & 0 & 0 & z w_{2} & 0 & 0 \\
0 & 0 & 0 & 0 & 0 & 0 & 0 & z w_{3} & 0 & 0 & 0
\end{array}\right]^{t} .
$$

The magnetic voltages on cores can be written as:

$$
\left[\boldsymbol{E}_{g}\right]=\left[\boldsymbol{R}_{g}\right] \cdot\left[\boldsymbol{\Phi}_{g}\right]-[\boldsymbol{Z w}] \cdot[\boldsymbol{i}],
$$

where $[i]=\left[i_{1}, i_{2}, i_{3}\right]^{t}$ and $i_{1}, i_{2}, i_{3}$ are the currents flowing through the windings.

The equations of magnetic tensions of the circuit can be written as:

$$
[\boldsymbol{p o l}] \cdot\left[\boldsymbol{E}_{g}\right]=[0]
$$

At the same time, voltage equations for windings (equivalent to Equation [4]) can be written (after integrating) as:

$$
\left[\boldsymbol{L}_{\boldsymbol{\sigma}}\right] \cdot[\boldsymbol{i}]+[\boldsymbol{Z} \boldsymbol{w}]^{t} \cdot\left[\boldsymbol{\Phi}_{\boldsymbol{g}}\right]=\left[\begin{array}{l}
\int u_{1} \mathrm{~d} t \\
\int u_{2} \mathrm{~d} t \\
\int u_{3} \mathrm{~d} t
\end{array}\right]=\left[\boldsymbol{E}_{\boldsymbol{C}}\right],
$$

To Equations (14) and (16) we incorporate Equation (10) that expresses the branch fluxes 
$\left[\Phi_{g}\right]$ through the loop fluxes $\left[\Phi_{o}\right]$. Then, from the Equation (16) we calculate currents $[i]$ and insert them into the Equation (14). What we get from Equation (15) is:

$$
\begin{gathered}
\left\{[\boldsymbol{p o l}] \cdot\left[\boldsymbol{R}_{g}\right] \cdot[\mathbf{p o l}]^{t}+[\boldsymbol{p o l}] \cdot[\boldsymbol{Z w}] \cdot\left[\boldsymbol{L}_{\sigma}\right]^{-1} \cdot[\boldsymbol{Z w}]^{t} \cdot[\mathbf{p o l}]^{t}\right\}\left[\boldsymbol{\Phi}_{o}\right]= \\
=[\boldsymbol{p o l}] \cdot[\boldsymbol{Z w}] \cdot\left[\boldsymbol{L}_{\sigma}\right]^{-1} \cdot\left[\boldsymbol{E}_{\boldsymbol{C}}\right]=[\boldsymbol{P}] \ldots
\end{gathered}
$$

and:

$$
[\boldsymbol{i}]=\left[\boldsymbol{L}_{\sigma}\right]^{-1} \cdot\left\{\left[\boldsymbol{E}_{\boldsymbol{C}}\right]-[\boldsymbol{Z w}]^{t} \cdot[\boldsymbol{p o l}]^{t} \cdot\left[\boldsymbol{\Phi}_{o}\right]\right\} .
$$

Equations (17) and (18) are equivalent to Equations (8) and (9). Equation (17) as well as (8) have a symmetric matrix, obtained after reduction of the vector of currents [i].

Further considerations will be carried out with the denotations from the Equations (8) and (9), that correspond to equations $(17,18)$ :

$$
\begin{aligned}
& {[M]=[\mathrm{pol}] \cdot\left[\mathrm{R}_{\mathrm{g}}\right] \cdot[\mathrm{pol}]^{t},} \\
& {\left[\mathrm{~T}_{0}\right]=[\mathrm{pol}] \cdot[\mathrm{Zw}] .}
\end{aligned}
$$

The matrix $\left[\boldsymbol{T}_{0}\right]$ has dimensions $s \times f(s=7, f=3)$. The dimension $s$ is the number of independent loops from the system in Fig. 1. At the same time it is the number of unknowns of the loop flux method $[x]=\left[\Phi_{o}\right]$. When calculating with the finite elements method there is a very large number of unknowns in this method, i.e. $\left[\boldsymbol{X}_{12}\right]$ from the Equation (7). It should be noted that the columns of the matrix $\left[\boldsymbol{T}_{\mathbf{0}}\right]$ from equations $(5,7)$ and $(19)$ are not always independent from each other, i.e. they are not always mutually orthogonal. Using the GramSchmidt method of orthogonalization $[2,4]$ one can achieve mutual orthogonality of matrix columns $\left[\boldsymbol{T}_{\mathbf{0}}\right]$.

$$
\left[\boldsymbol{T}_{\mathbf{0}}\right]=[\boldsymbol{w}] \cdot[\boldsymbol{p}], \text { while }[\boldsymbol{w}]^{t} \cdot[\boldsymbol{w}]=[\boldsymbol{I}]_{f} .
$$

The matrix $[w]$ includes $f=3$ mutually orthogonal columns. The matrix $[p]$ is a permutation matrix with $f \times f$ dimensions, and $\left[\boldsymbol{I}_{f}\right.$ is the identity matrix.

The main matrix of the system (17) can be written as:

$$
[m g l]=[M]+[w] \cdot[p] \cdot\left[L_{\sigma}\right]^{-1} \cdot[p]^{t} \cdot[w]^{t} .
$$

We introduce a denotation:

$$
[\lambda]=[\mathbf{p}] \cdot\left[\mathbf{L}_{\sigma}\right]^{-1} \cdot[\mathbf{p}]^{\mathbf{t}},
$$

i.e.

$$
[\mathbf{m g l}]=[\mathbf{M}]+[\mathbf{w}] \cdot[\lambda] \cdot[\mathbf{w}]^{t} .
$$

The matrix $\left[\boldsymbol{L}_{\boldsymbol{\sigma}}\right]$ has on its diagonal leakage inductance of windings, which were adopted as: 


$$
\operatorname{diag}\left(\boldsymbol{L}_{\boldsymbol{\sigma}}\right)=\left[\begin{array}{lll}
0.004 & 0.0018 & 0.0013
\end{array}\right]
$$

One can compare the eigenvalues of the loop flux matrix $[\boldsymbol{M}]$ and the main matrix $[\boldsymbol{m g} \boldsymbol{l}]$ of the system (17) (arranged in rows):

$$
\begin{aligned}
& {[\boldsymbol{M}] \rightarrow\left[\begin{array}{lllllll}
0.69783 & 1.6748 & 4.7887 & 5.7198 & 9.6157 & 11.387 & 57.683
\end{array}\right]} \\
& [\boldsymbol{m g l}]] \rightarrow\left[\begin{array}{lllllll}
0.75934 & 2.0638 & 6.2271 & 49.228 & 822.86 & 2016.3 & 24294
\end{array}\right]
\end{aligned}
$$

It can be seen that the ratio of the largest eigenvalue to the smallest one i.e. the condition number has been enlarged in the matrix $[\boldsymbol{m g l}]$, after the introduction of windings and leakage inductance $\left[\boldsymbol{L}_{\boldsymbol{\sigma}}\right]$ into the system:

$$
82.66=\frac{57.684}{0.69783} \rightarrow 31994=\frac{24294}{0.75934} .
$$

This implies a huge inconvenience in iterative calculations and threatens their stability. This occurs despite the fact, that the matrix $[\boldsymbol{m g} \boldsymbol{l}]$ is symmetric. The element which caused the deterioration of conditioning $[\lambda]$ is related to vectors which are columns of the matrix $[\boldsymbol{w}]$. To improve the condition number, one must separate the elements of the solution $[x]=\left[\Phi_{o}\right]$ of the system (17), associated with these vectors contained in the columns of the matrix $[w]$. This is the essence of the method presented in this article.

Therefore, we assume that the solution $[x]=[\Phi]$ of the system (17) can be written as:

$$
[x]=[w] \cdot\left[x_{0}\right]+[y] .
$$

We assume that the first part of the solution $[w] \cdot\left[x_{0}\right]$ belongs to the subspace created by vectors, which are columns in the matrix [w]. These vectors create the vector space $\Omega_{1}$. It has a small dimension $f=3$. The entire space which dimension is $s=7$, is denoted as $\Omega$. It contains the solution $[x]$. We assume that the part of the solution, denoted as $[\boldsymbol{y}]$ is orthogonal to the subspace $\Omega_{1}$ and belongs to the subspace $\Omega_{2}=\Omega \backslash \Omega_{1}$ :

$$
[w]^{t} \cdot[y]=[0]_{f=3} .
$$

For further calculations we assume that the vector $[P]$ of the right side of the Equation (17) is:

$$
[\boldsymbol{P}]=\left[\begin{array}{lllllll}
33010 & 9.5717 & -5995.1 & 6008 & -51149 & 18154 & 9.1574
\end{array}\right]^{t} .
$$

In order to calculate the vector $\left[x_{0}\right]$ from the formula (27) we substitute the solution $[x]$ from the formula (27) into Equation (17) and multiply the resulting equation from the left side by $[\boldsymbol{w}]^{t}$.

$$
\left\{[\boldsymbol{w}]^{t} \cdot[\boldsymbol{M}] \cdot[\boldsymbol{w}]+[\lambda]\right\} \cdot\left[\boldsymbol{x}_{0}\right]=[\boldsymbol{w}]^{t} \cdot[\boldsymbol{P}]-[\boldsymbol{w}]^{t} \cdot[\boldsymbol{M}] \cdot[\boldsymbol{y}] .
$$

Denoting:

$$
\left[\boldsymbol{M}_{0}\right]=[\boldsymbol{w}]^{t} \cdot[\boldsymbol{M}] \cdot[\boldsymbol{w}]+[\lambda],
$$

we have: 


$$
\left[x_{0}\right]=\underbrace{\left[M_{0}\right]^{-1} \cdot[w]^{t} \cdot[P]}_{[x 00]}-\underbrace{\left[M_{0}\right]^{-1} \cdot[w]^{t} \cdot[M]}_{[y 00]} \cdot[y] .
$$

Due to the size of the vector $\left[x_{0}\right]$ equal to $f=3$, the above calculation is fast and carried out by the non-iterative method (direct). The vector $\left[\boldsymbol{x}_{0}\right]$ from the formula (32) is substituted to the formula (27), on $[\boldsymbol{x}]$, and then to the Equation (17). The purpose is to calculate the vector $[\boldsymbol{y}]$. This way we obtain:

$$
\begin{aligned}
& \left\{[\boldsymbol{M}]+[\boldsymbol{w}] \cdot[\lambda] \cdot[\boldsymbol{w}]^{t}-([\boldsymbol{M}] \cdot[\boldsymbol{w}]) \cdot\left[\boldsymbol{M}_{0}\right]^{-1} \cdot\left([\boldsymbol{w}]^{t} \cdot[\boldsymbol{M}]\right)-[\boldsymbol{w}] \cdot[\lambda] \cdot\left[\boldsymbol{M}_{0}\right]^{-1} \cdot\right. \\
& \left.\cdot\left([\boldsymbol{w}]^{t} \cdot[\boldsymbol{M}]\right)\right\} \cdot[\boldsymbol{y}]=[\boldsymbol{P}]-[\boldsymbol{m g l}] \cdot[\boldsymbol{w}] \cdot\left[\boldsymbol{M}_{0}\right]^{-1} \cdot[\boldsymbol{w}]^{t} \cdot[\boldsymbol{P}] \Leftrightarrow[\boldsymbol{m a c}] \cdot[\boldsymbol{y}]=[\boldsymbol{b}]
\end{aligned}
$$

This is a linear Equation (1). Due to its large dimensions as for the finite element method, it should be solved by iterative methods. In the case of the finite element method, the calculation of the residue $\overrightarrow{\boldsymbol{r e s}}$ from the formula (2) for this equation involves operations on sparse matrices which are multiplied by vectors. Therefore, it is fast.

The example considered in Fig. 1 has small dimensions. Therefore one can compare the results of the presented method and direct calculations.

The results of direct calculations $[\boldsymbol{x}]=\left[\Phi_{o}\right]$ from the Equation (17) are applied to calculate the vector $[\boldsymbol{y}]$ by separating from $[\boldsymbol{x}]$ the components that belong to the subspace $\Omega_{2}$. In order to do this, the solution $[\boldsymbol{x}]$ should be multiplied by the matrix $[\boldsymbol{U}]$.

$$
[\boldsymbol{U}]=\operatorname{eye}(s)-[\boldsymbol{w}] \cdot[\boldsymbol{w}]^{\boldsymbol{t}} ;[\boldsymbol{y}]=[\boldsymbol{U}] \cdot[\boldsymbol{x}],
$$

where eye $(s)$ is the identity matrix of dimensions $s=7$. The vector $[y]$ calculated in this way is used for comparison with the vector $[y]$ calculated from Equation (33). Similarly, knowing $[\boldsymbol{y}]$ one can calculate $\left[\boldsymbol{x}_{\boldsymbol{o}}\right]$ with (32).

It can be compared with the results $[\boldsymbol{x}]$ of a directly solved Equation (17) by means of the multiplication:

$$
\left[x_{0}\right]=[w]^{t} \cdot[x] .
$$

Such verification is only possible thanks to small dimensions of the example considered in Fig. 1.

The matrix $[\boldsymbol{m a c}]$ of the equation system (33) is not symmetrical. The missing element $[\boldsymbol{d o d}]$ of its symmetrization is the transposition of the last component of the matrix [mac] in the formula (33):

$$
[\mathrm{dod}]=-[M] \cdot[w] \cdot\left[\mathrm{M}_{0}\right]^{-1} \cdot[\lambda] \cdot[w]^{t} .
$$

In the Equation (33) this element is multiplied by a vector of unknowns $[y]$. Due to the assumption (28), the vector $[\boldsymbol{y}]$ belongs to the subspace $\Omega_{2}$. In the same way also:

$$
[\text { dod }] \cdot[y]=[0]_{S=7} .
$$


Therefore, an additional element of [dod] does not contribute to the residuum $\overrightarrow{\boldsymbol{r e s}}$ (2) of the system of Equations (33) and therefore may be abandoned. After abandoning the element [dod], despite the asymmetry, the iterative methods can be used, e.g. the minimal residual method, suitable for symmetrical systems. This accelerates calculation process considerably.

The right side $[\boldsymbol{b}]$ of the system (33) also belongs to the subspace $\Omega_{2}$. It can be seen after multiplying the left side by $[\boldsymbol{w}]^{t}$ :

$$
[\boldsymbol{w}]^{t} \cdot[\boldsymbol{b}]=[\boldsymbol{w}]^{t} \cdot[\boldsymbol{P}]-\underbrace{[\boldsymbol{w}]^{t} \cdot[\boldsymbol{m g l}] \cdot[\boldsymbol{w}]}_{\left[M_{0}\right]} \cdot\left[\boldsymbol{M}_{0}\right]^{-1} \cdot[\boldsymbol{w}]^{t} \cdot[\boldsymbol{P}]=[0]_{f} .
$$

Taking into account Equations (23) and (31) we obtain a confirmation of the formula (38).

During the iterative process of solving the system of Equations (33), both the solution $[y]$ and the vector of residuals (2) should belong to the subspace $\Omega_{2}$, thus should be orthogonal to the vector-columns of the matrix $[\boldsymbol{w}]$. However, due to rounding errors, there may occur components that belong to the subspace $\Omega_{1}$. These errors will increase, because when calculating the residuum (2) they are multiplied by large eigenvalues of the matrix [mac], that correspond to the subspace $\Omega_{1}$.

They can cause a loss of stability of the minimal residual method, which manifests itself in a normal increase of the residue during iteration. In order to counteract, these components must be removed during iteration. For this purpose, the substitution by means of the matrix $[\boldsymbol{U}]$ from the formula (34) should be performed during iteration:

$$
[y]:=[U] \cdot[y] .
$$

Removing these components corresponds to the Chebyshev iterative method, carried out on error vectors corresponding to large eigenvalues. This was previously described as the combination of the Chebyshev method and the minimal residual method. However, it is much easier and faster.

In order to prove the assumption (28) of the vector $[y]$ belonging to the space $\Omega_{2}$, one must prove that the solution of the system (33) has this property.

For this purpose, we multiply the left side of the equation (33) by $[w]^{t}$. We use the Equations (38) and (31). We also use mutual orthogonality of the matrix $[w]$ columns (20).

$$
[\mathbf{w}]^{\mathbf{t}} \cdot([\mathbf{m a c}] \cdot[\mathbf{y}])=[\mathbf{w}]^{\mathrm{t}} \cdot[\mathbf{b}] \Rightarrow[\lambda] \cdot\left([\mathbf{w}]^{\mathrm{t}} \cdot[\mathbf{y}]\right)=[\mathbf{0}]_{\mathrm{f}} \Rightarrow \text { equation (28). }
$$

The matrix $[\mathbf{m a c}]$ of the system of Equations (33) is asymmetric. This does not prevent the use of iterative methods designed for symmetric systems to solve this system. This is because the solution $[\boldsymbol{y}]$ of this system belongs to the subspace $\Omega_{2}$, and the symmetry defect $[\boldsymbol{d o d}]$ (36) of this matrix [mac] relates to the subspace $\Omega_{1}$. The subspace $\Omega_{1}$ is disjoint with the subspace $\Omega_{2}$. These dependences can be presented by placing values and eigenvectors of these matrices in Tables 1 and 2.

As illustrated in Tables 1 and 2, the matrix $[\mathbf{m a c}]$ is taken from the Equation (33), the supplementary matrix to the symmetry $[\boldsymbol{d o d}]$ is taken from the formula (36). When comparing both tables it can be seen that the subspace $\Omega_{2}$, to which the solution $[y]$ of the system (33) 
belongs, is identical for each matrix. They contain the same eigenvalues and corresponding (placed in columns under the eigenvalues) eigenvectors. The condition number of the system (33) for calculations $[y]$ in subspace $\Omega_{2}$ amounts $49.232 / 0.75934=65$. The subspace $\Omega_{1}$, created by vectors that are the columns of the matrix $[w]$ from the Equation (20) for both the matrix of Table 1 and 2 has slightly different eigenvalues and eigenvectors. But in both cases it is the same subspace $\Omega_{1}$, obtained as $\Omega_{1}=\Omega \backslash \Omega_{2}$.

Table 1. Eigenvalues (in the first row) and, below them in the columns, eigenvectors of asymmetrical matrix $[\mathbf{m a c}]$ from the system of Equations (33)

\begin{tabular}{c|l|l|l|l|l|l}
\hline \multicolumn{5}{c|}{$\Omega_{2}$} & \multicolumn{1}{c}{$\Omega_{1}$} \\
\hline 0.75934 & 2.0638 & 6.2271 & 49.232 & \multicolumn{1}{c}{815.33} & 2000 & 24285 \\
\hline-0.33253 & -0.016104 & 0.47169 & 0.0013036 & 0.42165 & $-1.6865 \mathrm{e}-04$ & -0.69871 \\
\hline-0.47931 & 0.51316 & -0.32214 & 0.63495 & -0.0013169 & 0.006042 & $6.9107 \mathrm{e}-05$ \\
\hline-0.4682 & -0.25424 & -0.33787 & -0.31937 & 0.001261 & 0.70431 & $-2.7257 \mathrm{e}-05$ \\
\hline-0.4682 & -0.25424 & -0.33787 & -0.31937 & 0.001261 & -0.70984 & $-2.7257 \mathrm{e}-05$ \\
\hline-0.33253 & -0.016104 & 0.47169 & 0.0013036 & 0.39294 & $-1.6865 \mathrm{e}-04$ & 0.71522 \\
\hline-0.33253 & -0.016104 & 0.47169 & 0.0013036 & -0.81719 & $-1.6865 \mathrm{e}-04$ & -0.016606 \\
\hline-0.010494 & 0.77886 & 0.020925 & -0.62677 & $7.9325 \mathrm{e}-05$ & -0.0060384 & $1.7218 \mathrm{e}-07$ \\
\hline
\end{tabular}

Table 2. Eigenvalues (in first row) and, below them in the columns, eigenvectors of symmetrical matrix $[\mathrm{mac}]+[\mathrm{dod}]$

\begin{tabular}{c|l|l|l|l|l|l}
\hline \multicolumn{5}{c}{$\Omega_{2}$} & \multicolumn{1}{c}{$\Omega_{1}$} \\
\hline \multicolumn{1}{c|}{0.75934} & \multicolumn{1}{c}{2.0638} & 6.2271 & \multicolumn{1}{c}{49.232} & \multicolumn{1}{c}{807.86} & \multicolumn{1}{c}{1984} & 24275 \\
\hline 0.33253 & -0.016104 & -0.47169 & 0.0013036 & 0.42251 & $-9.1128 \mathrm{e}-04$ & 0.69868 \\
\hline 0.47931 & 0.51316 & 0.32214 & 0.63495 & $4.336 \mathrm{e}-16$ & $-4.1949 \mathrm{e}-17$ & $2.0375 \mathrm{e}-17$ \\
\hline 0.4682 & -0.25424 & 0.33787 & -0.31937 & 0.0014722 & 0.70711 & $3.1981 \mathrm{e}-05$ \\
\hline 0.4682 & -0.25424 & 0.33787 & -0.31937 & -0.0014722 & -0.70711 & $-3.1981 \mathrm{e}-05$ \\
\hline 0.33253 & -0.016104 & -0.47169 & 0.0013036 & 0.39382 & $-7.876 \mathrm{e}-04$ & -0.71524 \\
\hline 0.33253 & -0.016104 & 0.47169 & 0.0013036 & -0.81633 & 0.0016989 & 0.016563 \\
\hline 0.010494 & 0.77886 & -0.020925 & -0.62677 & $-7.9445 \mathrm{e}-18$ & $-6.4153 \mathrm{e}-19$ & $2.3809 \mathrm{e}-20$ \\
\hline
\end{tabular}

The scheme shown in Fig. 1, has small dimensions of the system of Equations (33), so direct methods should rather be used to solve it. The equations of type (33) obtained from the finite element method, however, have large dimensions and must be solved with the use of iterative methods, operating in the subspace $\Omega_{2}$.

\section{Conclusions}

Systems with magnetically coupled windings solved with the finite element method have an ill-conditioned skew-symmetric matrix [13]. The method proposed in the article is based on 
direct solving of a system with large eigenvalues of the problem. In this way the spectrum decreases (spectrum of eigenvalues), to the remaining components of the solution. This simplifies and accelerates the iterative process of obtaining the remaining components of the solution. A condition number understood as the ratio of the largest and smallest eigenvalues for the iterative method is therefore significantly improved. This is particularly important with a very large number of unknowns of the problem.

The proposed method was exemplified with the magnetic circuit with windings, solved by a circuital method. Due to small dimensions one could observe numerical effects of individual steps of the algorithm.

The application of the presented algorithm in the finite element method was performed in $[6-8,14]$. The 3 -column autotransformer with five windings on each column was investigated. Mono-harmonic calculations were performed for the given pulsations on complex numbers. An autotransformer impedance matrix was created. Having found eigenvalues and eigenvectors, the autotransformer inductance was identified [6-8].

It should be underlined that in space $\Omega_{1}$, solved with direct methods, there are not only the effects of the leakage inductance (including inductance in end winding connections in $2 \mathrm{D}$ calculations), of external connections $\left[\boldsymbol{L}_{\boldsymbol{\sigma}}\right]$, but also magnetic fluxes, calculated with the finite element method. They seem to be "selected" using vectors-columns of the matrix $[\boldsymbol{w}]$. It is evidenced by the formula (31) of matrix $\left[\boldsymbol{M}_{0}\right]$. Those chosen, additional components are equal to $[\boldsymbol{w}]^{t} \cdot[M] \cdot[\boldsymbol{w}]$.

\section{References}

[1] Demenko A., Wojciechowski R., Sykulski J., 2-D versus 3-D electromagnetic field modeling in electromechanical energy converters, IEEE Transactions on Magnetics, vol. 50, no. 2, pp. 897-900 (2014).

[2] Saad Y., Iterative Methods for Sparse Linear Systems, Second Edition, Society for Industrial and Applied Mathematics (2003).

[3] Traub J.F., Woźniakowski H., On the Optimal Solution of Large Linear Systems, Department of Computer Science Columbia University (1982).

[4] Higham N.J., Accuracy and Stability of Numerical Algorithms, Second Edition, Society for Industrial and Applied Mathematics (2002).

[5] Saad Y., Numerical Methods for Large Eigenvalues Problems, Second Edition, Society for Industrial and Applied Mathematics (2011).

[6] Gołębiowski M., The effect of the leakage inductance auto-transformers, which supply a multipulse rectifier systems on the network current distortion factor (dissertation in Polish), $\mathrm{PhD}$ Thesis, The Faculty of Electrical and Computer Engineering, Rzeszów University of Technology, Rzeszów (2010).

[7] Gołębiowski M., Mazur D., Calculating the main and leakage inductance matrix of the 3-column 15-winding autotransformer, Archives of Electrical Engineering, vol. 60, no. 3, pp. 215-222 (2011).

[8] Gołębiowski M., Mazur D., Measurement and calculation of 3-column 15-winding autotransformer, Archives of Electrical Engineering, vol. 60, no. 3, pp. 223-230 (2011).

[9] Jin J.M., The Finite Element Method in Electromagnetics, 3rd Edition, Wiley-IEEE Press (2014).

[10] Tomczuk B., Koteras D., Waindok A., 3D Field Calculations of the Modular Transformer Heating under High Frequency Operation, Proceedings of the XIX International Conference on the Computation of Electromagnetic Fields, Budapest, Hungary (2013). 
[11] Gołębiowski M., Gołębiowski L., Mazur D., Voltages in the Shaft of the Induction Motor in 3D FEM Formulation, IEEE International Symposium on Diagnostics for Electric Machines, Power Electronics and Drives, Valencia, Spain, pp. 142-145 (2007).

[12] Choi S.C.T., Minimal Residual Methods for Complex Symmetric, Skew Symmetric and Skew Hermitian Systems, arXiv: 1304.6782v2 [cs.MS] (2014).

[13] Nowak L., Field Models of Transients in Electromechanical Converters (in Polish), Poznan University of Technology Publishing House (1999).

[14] Berhausen S., Paszek S., Use of the finite element method for parameter estimation of the circuit model of a high power synchronous generator, Bulletin of the Polish Academy of Science, vol. 63, no. 3, pp. 575-582 (2015). 